\title{
EMD BASED SPEECH ENHANCEMENT USING SOFT AND HARD THRESHOLD TECHNIQUES
}

\author{
Rudresh $\mathrm{MD}^{1}$, Rajeshwari $K \mathrm{M}^{2}$, Sujatha $\mathrm{S}^{3}$, Suresh $\mathrm{M}^{4}$ \\ ${ }^{1}$ Associate Professor, Department of ECE, Institute Kalpataru Institute of Technology Tiptur, Karnataka, India \\ ${ }^{2} M$ Tech (Digital Communication), Department of ECE, CMRIT Bengaluru, Karnataka, India \\ ${ }^{3}$ Associate Professor, Department of TCE, CMRIT Bengaluru, Karnataka, India \\ ${ }^{4}$ Associate Professor, Department of ECE, Institute Kalpataru Institute of Technology Tiptur, Karnataka, India
}

\begin{abstract}
In last few decades many attempts have been made on speech signals to eliminates the noise. Purpose of use any speech enhancement algorithm is to eliminate noises in variety of environments; most prominent of which are telecommunication applications. The purpose of this paper is to development of a novel speech enhancement algorithm which offers superior noise reduction over current methods. This Research paper work demonstrates a novel time domain speech enhancement algorithm for speech signals called empirical mode decomposition (EMD). EMD decomposes the speech signal corrupted by noise signal into a finite number of band limited signals known as intrinsic mode functions (IMFs), using iterative procedure called sifting process. These IMFs are denoised by using two different techniques first method is IMFs thresholding method or direct method of speech enhancement and another technique is IMF frame based method are discussed in this work. Both the methods use soft and hard threshold techniques for denoising the IMFs which are obtained from EMD. These algorithms are implemented empirically by using MATLAB software on real time speech data. Experimental Results shows that IMFs frame based method superior than direct method this can be tested by adding noises with the different SNR values to the clean speech.
\end{abstract}

Keywords: Empirical Mode Decomposion (EMD), Intrinsic Mode Function (IMF), Hard Threshold, and Soft Threshold

$* * *$

\section{INTRODUCTION}

In many speech associated systems, the required signals are not directly obtained. It is contaminated with some unwanted interference noise bearing signals. These signals will degrade Perceptual Quality of the original speech signals therefore we need to suppress the noise or enhance the speech quality before transmission through telecommunication channels at the receiver end also we need to eliminate noise or enhance speech data once again because telecommunication channel also introduce some interfences while passing through them. Therefore we need to enhance speech signal otherwise this will degrade the performance of the communication devices which are used in multimedia applications such as cellphone and other multimedia devices etc. The various kinds of noise signal will disturb the original conversation. It might be a large band noise in terms of colored or white noise. it can take the form of fading noise or room reverberations. At a time, speech signal can be affected by multiple noise sources. The most common form of noise in signal processing and time series study was white noise. That is why in this primarily worried on this kind of noises [10].

There are several methods proposed for speech enhancement. The reported algorithm is divided into two main classes as parametric and non-parametric methods [10]. Parametric approaches assume a model for the process of signal generation. This model describes predictable structures and observable patterns in process .Noise reduction are performed depending on this priori information [10]. Since enhancement is depending on the parametric model, selection of the model is crucially important in those algorithms.

Non-parametric approaches simply need an estimation of the noise spectrum. This spectrum can be predicted by the pause periods where the speaker is silent (single channel) or from a reference source (multichannel) [15].

The speech enhancement methods can also be classified into single channel and multichannel approaches.

Single channel there is only one microphone and therefore only one noisy mixture which will just give spectral information. The noise spectrum has to be estimated from the pause period and the noise is regarded as stationary and uninterrupted. This makes the single channel speech enhancement challenging. That is why, the performance of the single channel techniques are limited [10].

In multichannel, multiple microphones are available in the environment, leading more noisy mixtures which exhibit the advantage of incorporating both spatial and spectral information. However, since multi microphones will come at an increased cost and may not be always available, the single channel speech enhancement always attracts attention [10]. 
Apart from above techniques lots of techniques are used for speech enhancement such as Fourier Transform based Techniques like short time Fourier transform, discrete cosine transform, wavelet transform they are all spectral domain methods and they applied on real time signals with some assumptions like system is linear even if the system is nonlinear and also assuming signals are stationary with the window even if the signals are nonstationary so these conventional methods are Not appropriate for nonlinear systems \& non stationary signals..So Norden E. Huang recently proposed time domain algorithm which is named as Empirical Mode Decomposition (EMD). Norden E. Huang s Work on EMD for signal is similar to the work of Sir Issac Newton on refraction of light.

\section{EAMPIRICAL MODE DECOMPOSITION}

A method that any complicated real time data set can be decomposed into a finite, small number of its constituents of components of data called Intrinsic Mode Functions (IMF's).

EMD is an important step to decompose the given signal into an IMF. For practical applications and pure research the data analysis was an essential part. It is easy to analyze the stationary processes and linear, but most of real life signals are non-stationary signals and nonlinear in the real world. It was not easy for analysis of such time varying data. Hence, Fourier spectral analysis is common and easy methods to provide distribution of global frequency energy. The entire duration the data set the spectrum gives exist of frequencies. However, the main aim of time frequency analysis to understand the rate of change of time with frequency content of data.

The time frequency (TF) represent, a two-dimensional function which indicates the signal content as function of the energy in both time and frequency, it was a strong tool in time-varying signals [18]. Simultaneously temporal and spectral information provided by $\mathrm{TF}$ representation. There are several number of TF representation ways and means for time domain signals, which are Wigner-expression, wavelet transform and Short time Fourier transform (STFT). Inside those, signal processing the wavelet and STFT have dominated in the time-frequency analysis.

The STFT represents the short time, snapshot like spectral representation in Fourier analysis the window-width will have limited time. It was simply obtained by sliding as selected size window with time axis and applying FT in each segment. Therefore, in case of non-stationary signals, the STFT has limited usage. The wavelet transform was the most common and currently used time frequency approaches.

For analyses non-stationary and nonlinear recently technique called as Hilbert Huang HHT [14]. It is a mixture Empirical Mode Decomposition (EMD). The main source in HHT was the EMD which reduces the signal into different kinds of frequency character, is named as intrinsic mode function (IMFs), this also diverges the problem of frequency change sharply in the originals signal. IMF gives the identification of instantaneous frequency. Thus they are extremely fit for Hilbert transformation. Once the IMF is found, HT may be applying for every IMF determines the time frequency component. Meanwhile EMD was introduced and implemented for non-stationary signal, for the different types of data, therefore frequently provides a superiority and efficiency.

\subsection{Intrinsic Mode Functions}

EMD algorithm is used to reduce any original signal $s(t)$ into a band limited function, with mean zero oscillating component, called IMFs [1].

Basic two conditions for satisfying IMF are

- Mean envelope of IMF should be zero.

- The zero crossing rate and local extreme should be equal to zero or at least differ by one.

First condition is same as Gaussian process used in narrow band. Second condition was induced necessary IF is not having proper fluctuation as persuaded by symmetric wave [1]. IMF is included as it significant mode of oscillation in data. Every cycle of IMFs, defined by zero crossings, the oscillation in any complex waves are allowed and only single mode is allowed. Narrow band signal are not restricted in the IMF, it may be frequency or amplitude modulation, and it may be non-stationary. Figure 1 shows typical IMF can be observed. To find the IMFs and eliminating data from uppermost oscillating components one by one is known as sifting process.

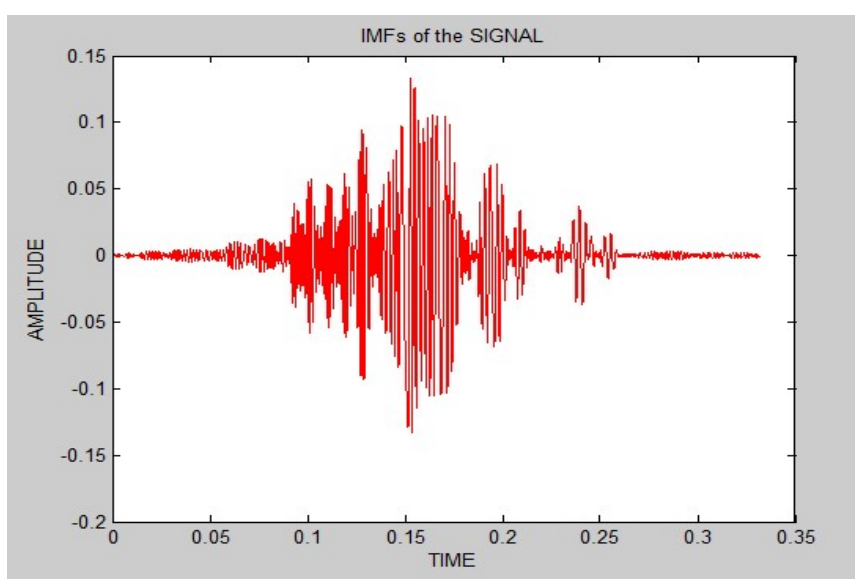

Figure 1. An IMF with same numbers of zero crossings and extreme, of envelopes with respect to zero mean.

\subsection{Sifting Process}

To implement the EMD algorithm various methods have been introduced, the first algorithm was called as sifting process. This process was easy and simple. Figure 2 shows the flow of sifting Process of EMD algorithm. And figure 4 shows the flowchart of EMD algorithm

1. Find the extreme of both minima and maxima of $S(t)$.

2. Produce lower $1(\mathrm{t})$ and upper envelopes $\mathrm{u}(\mathrm{t})$ by interpolating the both minima and maxima points.

3. Find local mean, $\mathrm{ml}(\mathrm{t})=[\mathrm{l}(\mathrm{t})+\mathrm{u}(\mathrm{t})] / 2$. 
4. If IMF should have mean value zero, then $\mathrm{h} 1(\mathrm{t})$ is obtained by subtracting $m_{1}(t)$ from the signal $s(t)$.

5. Then find the component $\mathrm{h}_{1}(\mathrm{t})$ is an IMF or not.

6. Else, consider $\mathrm{h}_{1}(\mathrm{t})$ as a fresh data and recap from 1 to 6 till an IMF is obtain [1].

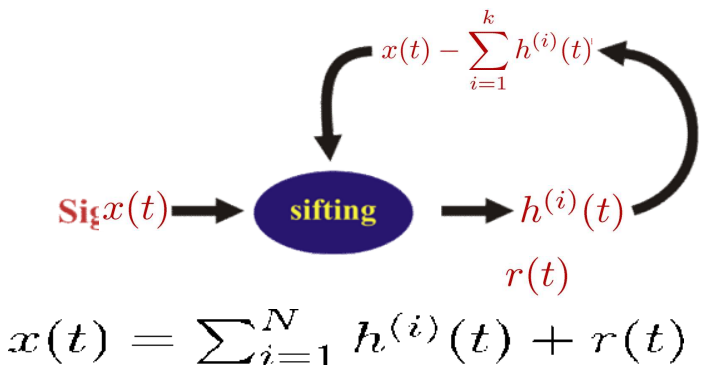

Figure 2. Working of sifting process

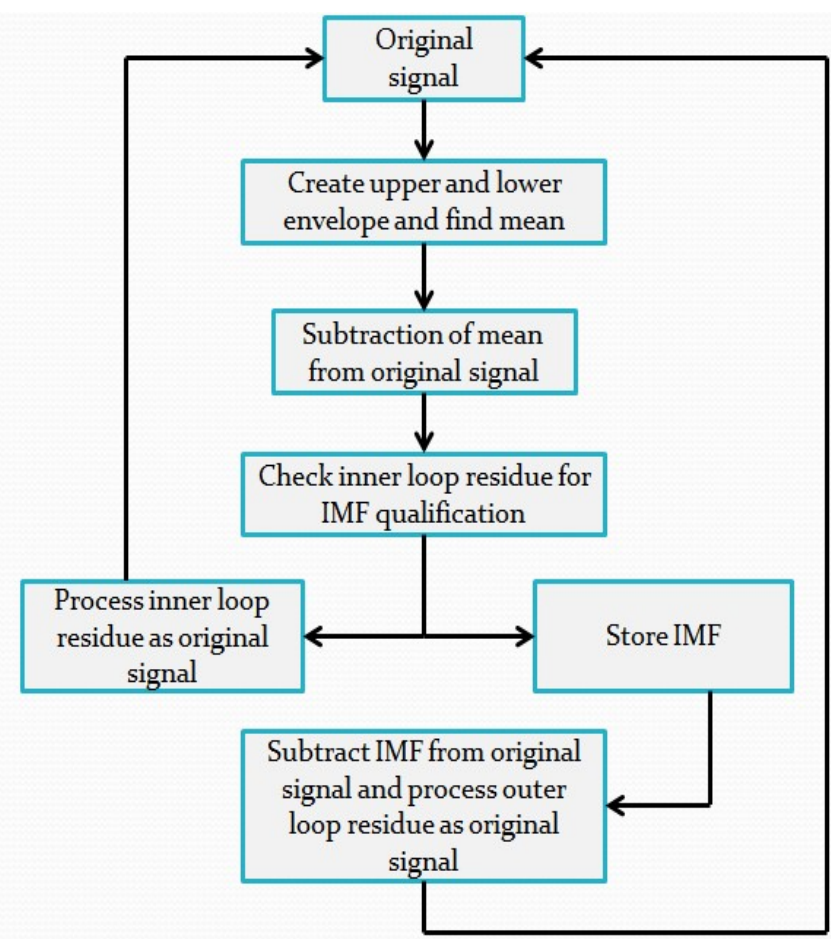

Figure 3. Flow chart of empirical mode decomposition

\section{EMD BASED SPEECH ENHANCEMENT}

\section{TECHNIQUES}

The empirical mode decomposition (EMD) is a recently developed new effective data analysis technique for nonstationary signals and nonlinear systems has made an effective track for enhancement of speech studies [15].

EMD is data-adaptive decay techniques with which any composite signal may be decayed into mean zero oscillating components and IMFs. Latest studies have shown that with $\mathrm{EMD}$, it is able to effectively remove noise sources from the noisy speech IMFs.

Soft thresholding strategy proposed a powerful process for eliminating the noise datas from noisy speech while concentrating on original speech. Since the signal dominant frames are not threshold, the algorithm enables seven signals with highestSNRs is managed successfully, anyway most of the reported approaches are fail to hold input SNR .Still, this is also a drawback, since it is not able to efficiently eliminate the noise source that is surrounded in higher energy speech signal. Since, categorizations of the frequency frames depend on the global noise variance, noise contain frames a recognized as signal frames due to variations in noise variance of frequency. As a result, the residual noise frames from both signal and noise dominant frames are result in an irritating musical noise.

In addition to this, another disadvantage of the previous method comes from the categorization in spectral domain. Since the DCT coefficients of every frame are distributed into 8 frequency bins, forming 8 sub-bands, we may have a sharp increase between a threshold noise dominant frequency bin and a non-threshold signal dominant frequency bin in the spectral domain. This also results in an irritating effect in the musical noise. All these drawbacks can be significantly reduced with the proposed EMD based soft thresholding strategy. To show a new speech enhancement technique based on EMD with soft thresholding process. The projected system significantly removes the noise. Meanwhile the extraction of IMFs relies on frequency characteristics; the IMFs with higher index contain lower frequency components. This property helps the noise and speech components to be roughly separated in terms of frequency and to dominate in different IMFs. In instance of white noise, the noise signal is dominate in first few IMF and the speech signal is dominate in the later IMFs. Therefore, the noise parts that are embedded in speech signals can also be extracted and threshold. In order to identify the noisy frames in an efficient way, the projected system also contains an alteration in the soft thresholding approach and a precise approach for every IMF of noisy speech.

EMD was a powerful tool recently developed for analysis data for non-stationary and nonlinear for speech enhancement [15]. The complex signal can be decompose into zero mean oscillating components by the data-adaptive decomposition method in the EMD is the intrinsic mode functions (IMFs). The noise components can easily and successfully remove the noisy speech component from the IMFs [1].

Soft thresholding is mainly concentrated on noise dominated frames in speech signal. Signal dominant frames are not threshold, to process efficiently in the algorithm enables with high SNRs, where the most of the method have fail to hold the input SNR. Hence, it is one of the drawbacks; it was not able to eliminate noise components effectively are entrenched at higher energy of speech signal [1]. Depending on variance of frequency classified as, noise frame from noise variance and speech frame from speech variance.

Disadvantage of previous methods is divided based on spectral domain. Each coefficients of DCT frame was divided into 8 frequency bands which form 8 sub-bands; it will increase sharply between a noise dominant thresholds frequency band and a signal dominant non-threshold frequency band in the spectral domain. This drawback will reduce by the proposed EMD based soft thresholding technique. 
A novel speech enhancement approach method is applied by soft thresholding algorithm with EMD. The noise is effectively removed by this proposed method. The extraction of frequency characteristics based on the IMFs relies. A lower frequency component is present in the higher index of the IMFs. In the algorithm, speech and noise are to be separated as frequency of IMFs. If it is white noise, the first few IMFs contain noise components dominated. The speech components are in the later IMFs. Thus, the speech signal contains the noise parts will also threshold and extracted.

\subsection{EMD based Soft Thresholding}

Let $\mathrm{Y}$ refer to the coefficients of a wavelet dimension of the noisy mixture signal and $\mathrm{T}$ be the threshold value for the Denoising strategy. The Soft thresholding arrange any coefficient with absolute value equal or less the threshold to zero and subtract threshold value from other coefficients [5].

$$
\mathrm{Y}=\left\{\begin{array}{cc}
\operatorname{sgn}(Y)(|Y|-|T|) & \text { if }|Y|>T \\
0 & \text { if }|Y|<T
\end{array}\right.
$$

Where $\mathrm{Y}$ denotes the threshold coefficients. It can be observed that the soft thresholding algorithm removes more noise components than the hard thresholding algorithm [18]. However in soft thresholding the amount of the signal degradation is also higher. Therefore, the thresholding strategy should be selected depending on the subjective and objective perspectives.

\subsection{EMD based Hard Thresholding}

Hard thresholding sets any coefficient whose absolute values is equal or less to the threshold zero [5],

$\mathrm{Y}= \begin{cases}Y & \text { if }|Y|>T \\ 0 & \text { if }|Y|<T\end{cases}$

Where the value of $\mathrm{T}$ is related with the estimated standard deviation of the noise signal $\sigma$ and may change depending on the proposed algorithm. Dohono has suggested the following formula for its value [5];

$\mathrm{T}=\sigma \sqrt{2 \log (\mathrm{n})}$

Due to the EMD efficiency in decomposing the nonstationary signal in terms of zero-mean oscillating components; with well-behaved instantaneous frequencies, it has been adapted almost all kinds of data analysis always verify its efficiency. Therefore EMD is a novel and effective path from any signal processing research areas. Speech processing is one of these fields that EMD has successfully been applied to. Due to this reason EMD algorithm applied to Speech Enhancement. this paper work proposes the two techniques of Speech Enhancement s they are
$>$ IMFs Threshold Technique of Speech Enhancement.

$>$ IMFs Framed Thresholding Technique

\subsection{IMFS THRESHOLDING TECHNIQUE OF SPEECH ENHANCEMENT}

Speech Enhancement is a traditional problem in signal Processing fields, Particularly in the case of AWGN (additive white Gaussian noise) recently different noise reduction techniques have been proposed for enhancing speech quality these methods works good only when noise estimation is available. However, these techniques are not working effectively when noise is difficult for estimating. Linear methods are widely used example for technique is Wiener filtering are used because linear filters are easy to design and implement. The linear techniques are not so effective for signals contains impulses or sharp edges with short in duration. Furthermore, real life signals are nonstationary in nature. In order to overcome above said short comings, nonlinear techniques were proposed and especially these based on principle of wavelet thresholding. The idea behind wavelet thresholding depends on the assumption of magnitudes of signal dominate the noise magnitudes in a wavelet representation, so that wavelet coefficients can be set to zero if their magnitudes are less than a predetermined threshold. In the wavelet approach its basis functions are not fixed, and, thus, do not match all real life signals. Recently, a new data-driven technique, referred to as Empirical mode decomposition (EMD) has been propesd by scientist name Huang for analyzing non-stationary data and nonlinear system. The advantage of the EMD algorithm is that its basis functions are derived from the original signal itself. Hence, the analysis of data is adaptive in contrast to the conventional methods, where the basis functions are fixed.

The Block Diagram for Direct IMFs thresholding Approach is shown in figure 4, In an IMFs thresholding Approach noisy Speech is first passed through the EMD Algorithm. The outputs of EMD are various intrinsic time scales of the signal, called intrinsic mode functions (IMFs), these IMFs are denoised by using hard threshold techniques or soft threshold techniques by calculating threshold value by using equation (1) and (2) and then these denoised IMFs are combined to get Enhanced Speech. In this method soft thresholding method gives better results than hard threshold techniques but draw back associated with both the methods is both methods eliminate noise completely but along with noise, speech data can also get eliminated this will degrade the quality of speech so we need to eliminate only noise by retaining the speech useful information this can be achieved by framing the IMFs and then thresholding this can be discussed by next method explained below

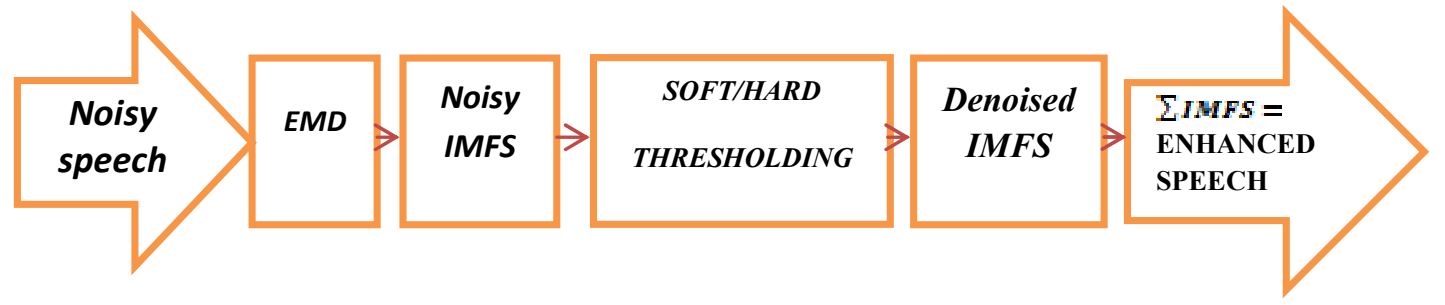

Figure 4. The block diagram for Direct IMFs thresholding Approach 


\subsection{IMFS Framed Thresholding Techniue}

The Block Diagram of the Proposed IMF frame based thresholding method is shown in figure..In IMFs framed threshold Technique instead of denoising the each IMFs which are obtained from EMD algorithm, each IMFs are segmented into number of frame according to frame duration and sampling frequency. These segmented individual frames are classified into noise dominented frame and speech dominented frame by using equation (5) and (6).

If the classified frames are belongs to noise dominented category those frames are threshold by using hard or soft threshold technique otherwise frames are belongs to speech dominented frame category hence no need to threshold leave it as it is. Similar experiment is repeated for all IMFs and then these IMFs are combined to get enhanced speech. Compared to first method this method gives better results because first two three IMFs contains much noise than speech, if we use direct method by fixing only one threshold value for entire IMFs then we can loss speech part while eliminating noise part.

The frames are categorized into signal dominant or noise dominant. Depending on its speech and noise energy distribution. The noise power related to specific frame can be defined by the equation for the ith frame is

$$
\frac{1}{64} \sum_{k=1}^{64}\left|X k^{i}\right|^{2} \geq \sigma_{n}^{2}
$$

Where $\sigma_{n}^{2}$ denotes the variance of the noise and $X k^{i}$ is the $k^{\text {th }}$ coefficient of the $i^{\text {th }}$ frame was identified as signal dominant else noise dominant. The signal dominant frames is not threshold, it was possible to reduce the speech signal for high SNR. If it is noise frame the coefficients values are arranged in ascending order and apply the thresholding techniques.
The classification of frames was one of major points of hard and soft thresholding algorithm. The reason for classification is to remove the noise source without reducing original speech source. Soft thresholding process is related in wide ranges of SNR is directly apply for the IMF of noisy speech is gets fail. The First IMFs will have various speech and noise variance. Second, the variance of the IMF frames will have more variations than the noisy speech frames, due to decomposition. The noise variance of every IMF is defined discretely and bound for frame categorization of larger value. This assures the entire noisy components are threshold. A new boundary relies that a frame may be determine as noise frame, if speech power within that frame was lesser than the noise power. If the speech and noise power is equal and independent then noise variance will be twice and equal to variance of a IMF frame. Therefore frame will be twice the estimated global noise variance this is said to $i^{\text {th }}$ frame [13];

$\frac{1}{64} \sum_{k=1}^{64}\left|X k m^{i}\right|^{2} \geq 2 \sigma_{n m}{ }^{2}$

\subsection{Variance of the IMFS}

The IMF plays an important role in finding of variance in every IMF. Soft thresholding algorithm estimates variance of EMD, the IMFs is classified as $4 \mathrm{~ms}$ frames and variance of every frame were arranged in variance array and then these variance are arranged in ascending order, Therefore the lowest variance values will have the speechless parts and high ranges having speech part more compare to noise. The IMFs of noise variance is estimated by speechless parts of the array. Figure 6 shows plot of the IMFs variance plots. The plots show that the first 3 IMFs contain the noise signals than speech signals. The remaining IMFs contain speech signals, with small amount of noise. The noise signal is removed by the specific noise variance and using the soft thresholding process [1].

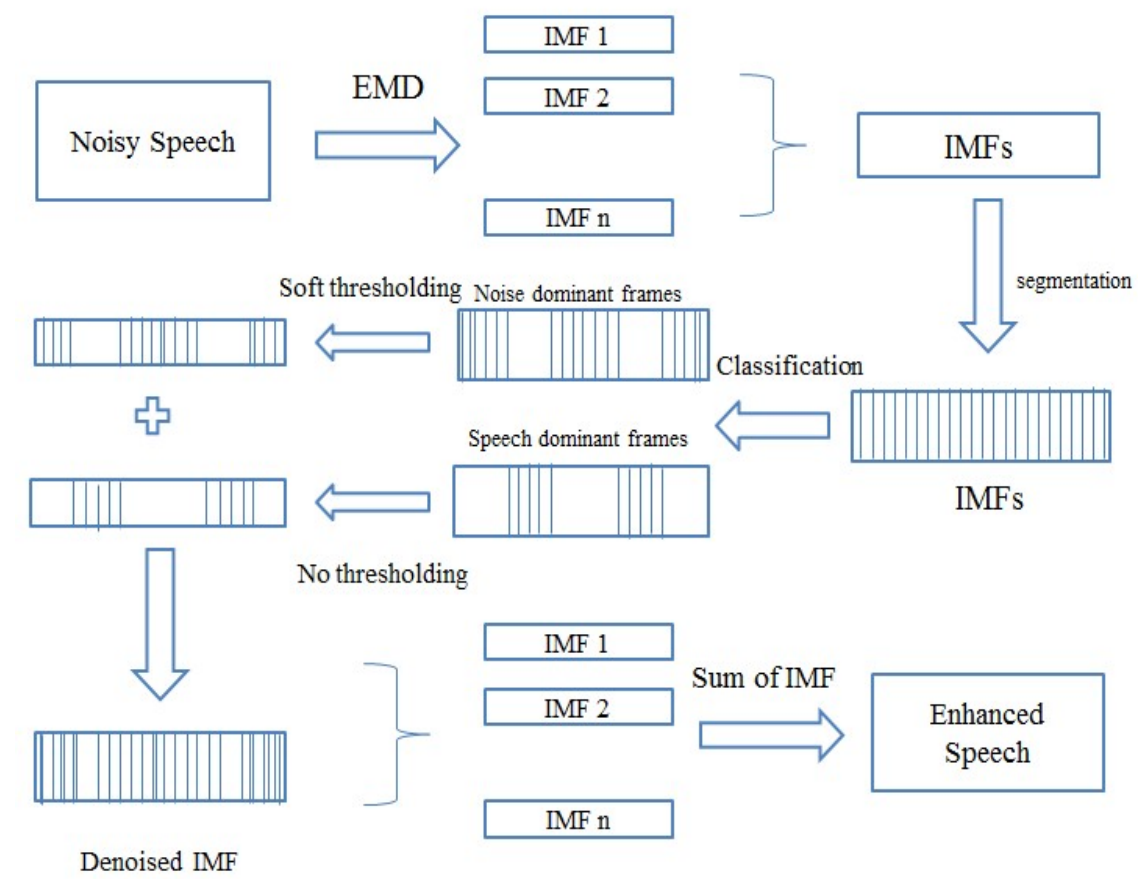

Figure 5. The Block Diagram of the Proposed IMF frame based thresholding method. 


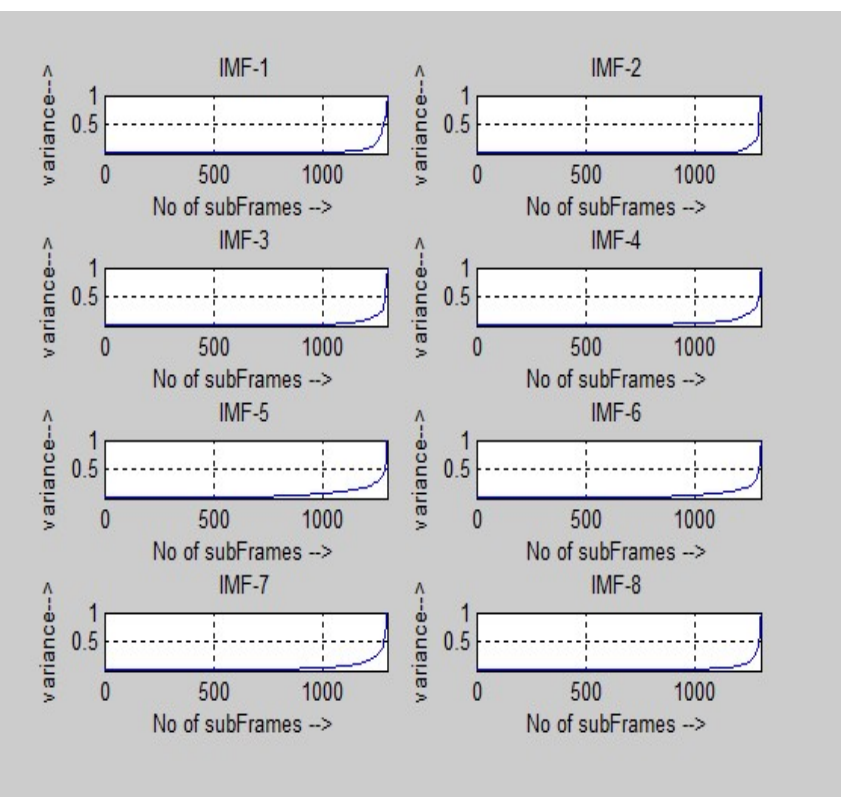

Figure 6. Sorted variance array with $4 \mathrm{~ms}$ frame duration for the first 8 IMFs of a noisy speech at $15 \mathrm{~dB}$ SNR

\section{EXPERIMENTAL RESULTS}

The proposed algorithms tested and demonstrated by using MATLAB software. To demonstrate the working of a direct and IMF frame threshold based methods we considered the sample speech data of TIMIT Database to measure the effectiveness of algorithms we plotted the spectrogram for original data and reconstructed speech data which is obtained by combining the denoised IMFs

Figure 6.1 illustrate the clean speech signal along with its spectrogram and figure 6.2 illustrate noisy at $5 \mathrm{~dB}$ SNR for the female voice "she had a your dark suite in greasy wash water all year" from sample data of TIMIT speech database is considered along with its spectrogram. Figure 6.3 and 6.4 illustrate the enhanced speech for female using the projected algorithm with soft and hard thresholding respectively along with its spectrogram. Figure 6.5 and 6.6 illustrate the enhanced speech for female using direct process algorithm with soft and hard thresholding respectively along with its spectrogram. The obtained IMFs in the algorithm were divided into $4 \mathrm{~ms}$ frames and each frame having 64samples of sampling frequency $16 \mathrm{~K} \mathrm{~Hz}$.

It is observed that the given scheme was effective in removal of noise and extremely superior to other reported methods. With the direct method there was a reasonable enhancement in the noisy speech signal. Although the noise components are successfully removed for wide range of frequencies, the remaining noise components in the enhanced signal can easily be observed. Whereas the proposed algorithm removes the remove the noise signals efficiently, while successfully speech signal is kept.

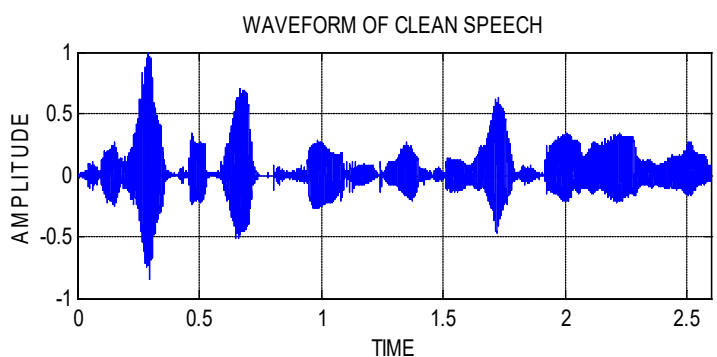

SPECTROGRAM OF ORIGINAL SPEECH WAVE

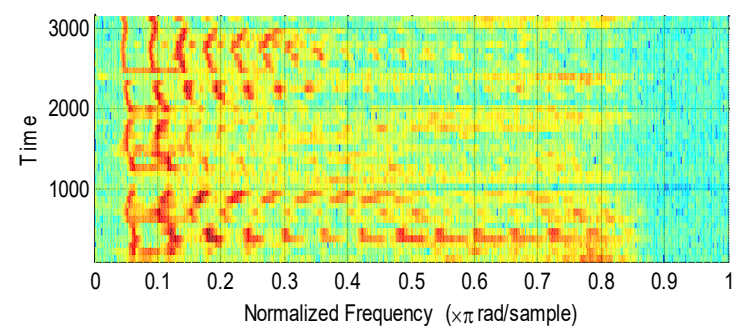

Figure 6.1. Plot of original speech and its spectrogram
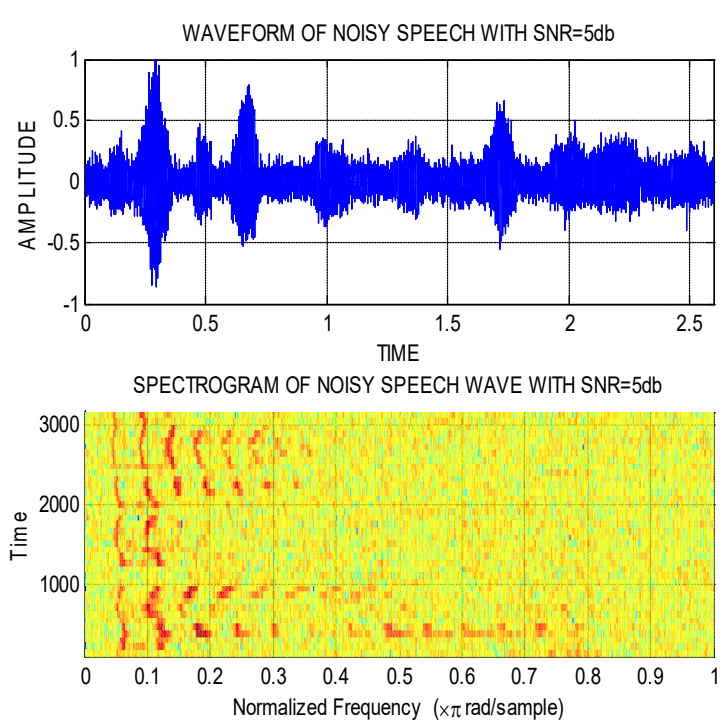

Figure 6.2. Plot of original speech with additive white Gaussian noise of $\mathrm{SNR}=5 \mathrm{~dB}$ and its spectrogram
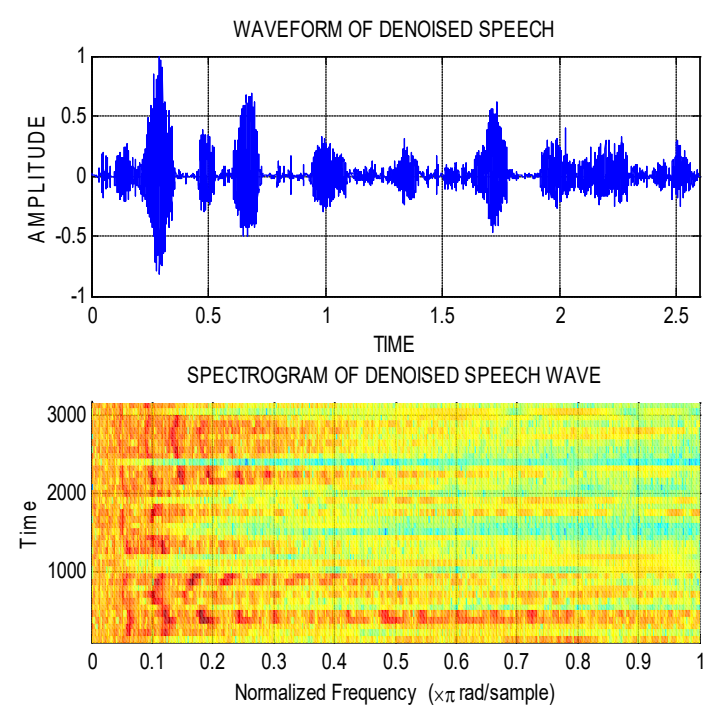

Figure 6.3 Plot of Enhanced speech by denoising using EMD based soft thresholding with framing for $\mathrm{SNR}=5 \mathrm{DB}$ 


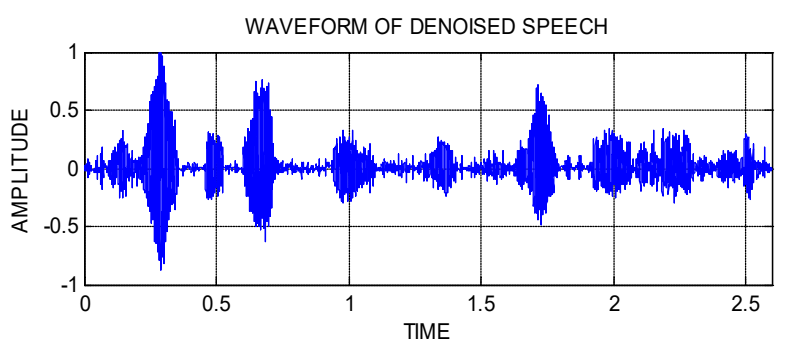

SPECTROGRAM OF DENOISED SPEECH WAVE

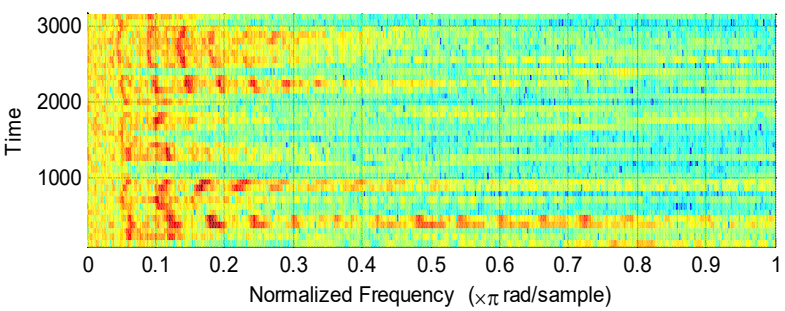

Figure-6.4.Plot of enhanced speech by denoising using

EMD based hard thresholding with framing for $\mathrm{SNR}=5 \mathrm{~dB}$

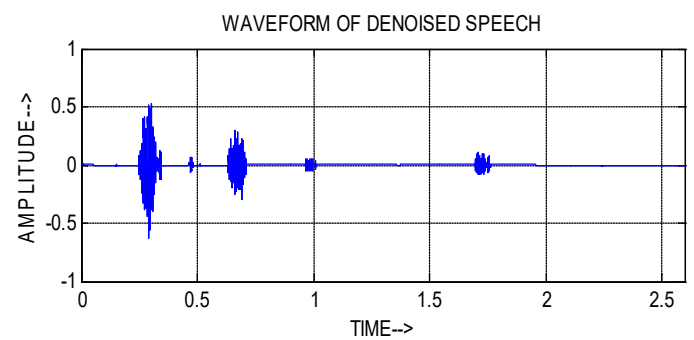

SPECTROGRAM OF DENOISED SPEECH WAVE

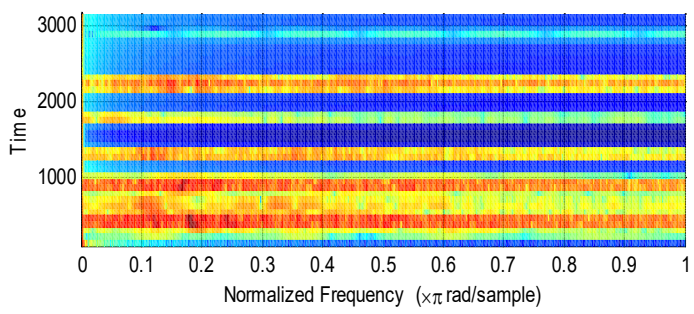

Figure 6.5. Plot of enhanced speech by denoising using EMD based soft thresholding without framing for $\mathrm{SNR}=5 \mathrm{~dB}$ (direct method)

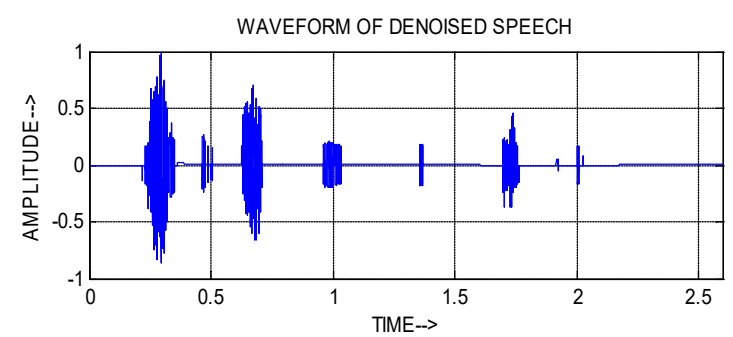

SPECTROGRAM OF DENOISED SPEECH WAVE

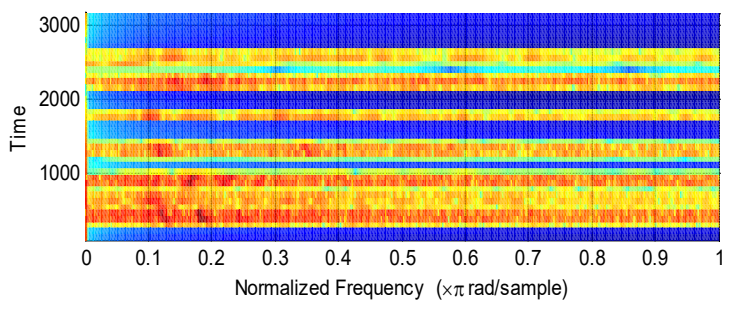

Figure 6.6 Plot of enhanced speech by denoising using EMD based hard thresholding without framing for $\mathrm{SNR}=5 \mathrm{~dB}$ (direct method)

\section{CONCLUSIONS}

In this paper work correspondence, we propose different speech Enhancement techniques such as IMF based method and IMF frame based techniques which are based on time domain algorithm Empirical Mode Decomposion (EMD) which is decomposing the signal into number of components in time domain. An experimental result shows that time domain EMD methods are also useful for improving the intelligibility and perceptual quality of a noisy speech signal, similar to Fourier Transform based methods, which eliminates the noisy signal from desired signal in frequency domain. This work also successfully demonstrated that IMF based Hard and soft threshold Techniques and also IMF frame based Hard and soft threshold Techniques, the results of above said methods shows, it is also possible to eliminate the noise from speech signal by using simple hard and soft thresholding methods these are widely used signal thresholding techniques, basically used with the algorithms of compressing the signals.

\section{REFERENCES}

[1] J. J. Soraghan and N. Chatlani, "Adaptive Empirical Mode Decomposition for Signal Enhancement with Application to Speech," In the National Taiwan Normal University, November 27, 2009.

[2] M. E. Hamid, K. Hirose, M. K. I. Molla, and S. Das, "Speech Enhancement Using EMD Based Adaptive Soft-Thresholding (EMD-ADT)," International journal of signal processing, Image Processing and Pattern Recognition, Vol. 5, No.2, June, 2012.

[3] N.E. Huang, Z.Shen,S.R. Long, M.C. Wu, "The Empirical Mode Decomposition andthe Hilbert Spectrum For Non-Linear And Non Stationary Time Series Analysis," In Proc. R. Soc., London, A454, Pp. 903-995, 1998.

[4] A. Bouzid and N. Ellouze, "Empirical Mode Decomposition of Voiced Speech Signal". IEEE Trans. FirstInternational Symposium on Control, Communication and Signal Processing. 2004, Pp. 603606.

[5] M. A. Kabir and C. Shahnaz, "Comparison of ECG Signal Denoising Algorithms in EMD and Wavelet domains", IJRRAS 11 (3), June 2012.

[6] Erhandeger, "Noise-Thresholding with Empirical Mode Decomposition for Low Distortion Speech Enhancement", In the University of $R$ Tokyo, February 2008.

[7] K. Yannis, and Stephen, "Enhanced Empirical Mode Decomposition Using A Novel Shifting Based Interpolation Points Detection". IEEE Workshop On Statically Signal Processing,2007. Pp. 725-729.

[8] J. S. Choi, "A Spectrum Recovery Algorithm using Signal-to-Noise Ratio classification for Noise Reduction", International Journal of Multimedia and Ubiquitous Engineering, Vol 8, No 4, July, 2013.

[9] D.G. Manolakis and J.G. Proakis. "Digital Signal Processing: Principles, Algorithms, and Applications", Prentice-Hall, volume 1, 3rd edition, 1996.

[10] T. L. Huang, W. Ren and M. L. Lou, "The Orthogonal Hilbert-Huang Transformand its Application in 
Earthquake Motion Recordings Analysis", $14^{\text {th }}$ World Conference on Earthquake Engineering, October 17, 2008.

[11] V. Sunnydayal, N. Sivaprasad, and T.K. Kumar. "A Survey on Statistical Based Single Channel Speech Enhancement Techniques", International Journal of Intelligent Systems and Applications, 2014.

[12] K. Khaldi. "Speech Enhancement via EMD", EURASIP Journal on Advances in Signal Processing, 2008.

[13] M. K. I. Molla, S. Das, M. E. Hamid and K. Hirose. "Empirical Mode Decomposition for Advanced Speech", Journal of signal processing, Vol 17, No 6, pp.215-229, November 2013.

[14] Dr. K. J. Sankar, Mr. S N. Rao and Dr. C.D Naidu. “An improved speech De-noising method based on empirical mode decomposition", IOSR Journal of VLSI and Signal Processing (IOSR-JVSP) Volume 5, Issue 2, Ver. I (Mar. - Apr. 2015), PP 01-09, 03/2015 $04 / 2015$.

[15] S. S. Nidhyananthan,K. R. S. Shantha, and A. A. Prakash. "A review on speech enhancement algorithms and why to combine with environment classification", International Journal of Modern Physics C, 2014.

\section{BIOGRAPHIES}

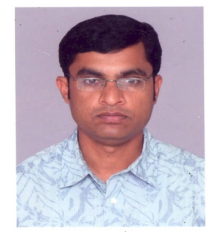

Rudresh M D, Associate Professor of Department of Electronics and Communication Engineering, Kalpataru Institute of Technology Tiptur, He has 15 years of teaching experience .He guided more than 15 projects for $\mathrm{PG}$ and $\mathrm{UG}$ Students from past 10 years. His areas of interest are DSP, Speech Processing, Image Processing, Embedded System, and Biomedical Signal Processing and have depth knowledge of microprocessors, Microcontrollers and DSP processors. Currently pursuing Ph D Programme at JNTU Hyderabad.

Email ID: rudresh_md@rediffmail.com

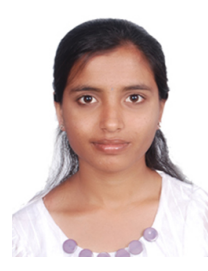

Rajeshwari K M Currently Pursuing M Tech (Digital Communication) at CMR Institute of Technology, Bengaluru. Her areas of interests are Embedded and DSP. Email ID: rajikm080@gmail.com

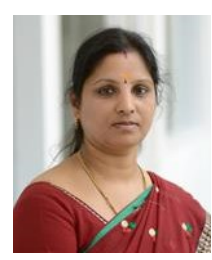

Sujatha S, Associate Professor of Department of Telecommunication Engineering, CMR Institute of Technology, Bengaluru. Her areas of interest are Analog electronics, of interest are Digital electronics, Microcontroller, MDSP430, Microwave and Radar Transmission line and wave guide, OCN, Embedded System and Wireless communication, CMOS and VLSI Design, OFDM. Her area of research Optical fiber Communication and Wireless Communication. Currently pursuing $\mathrm{Ph} \mathrm{D}$ at govt. Engg College, Pondicherry.

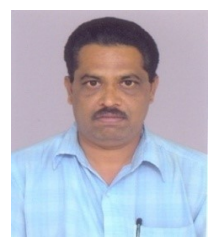

M. Suresh was born in India on October $28,1967 . \mathrm{He}$ received the $\mathrm{BE}$ degree in instrumentation at Siddaganga Institute of Technology, Tumkur, Karnataka and ME degree in electronics from the BMS College of engineering, Bangalore, India in 1991 and 1994 respectively. Currently he is Associate Professor with Electronics \& communication Department, Kalpataru Institute of Technology, Tiptur, India. Pursuing Ph D programme at Jawaharlal Nehru Technological University, Kukatpally, Hyderabad, and Telagana. His research interests include biometrics, speech signal processing. 